\title{
Sets with doubleton sections, good sets and ergodic theory
}

\author{
by \\ A. Kłopotowski (Paris), M. G. Nadkarni (Mumbai), \\ H. Sarbadhikari (Calcutta) and S. M. Srivastava (Calcutta)
}

\begin{abstract}
A Borel subset of the unit square whose vertical and horizontal sections are two-point sets admits a natural group action. We exploit this to discuss some questions about Borel subsets of the unit square on which every function is a sum of functions of the coordinates. Connection with probability measures with prescribed marginals and some function algebra questions is discussed.
\end{abstract}

Introduction. Given non-empty sets $X$ and $Y$, a subset of $X \times Y$ is called a matching if it is the graph of a one-to-one map of $X$ onto $Y$. The question as to which subsets $S$ of $X \times Y$ contain a matching has been of combinatorial and set-theoretic interest [12]. By a theorem of D. König [11], if each section $S_{x}=\{y \in Y:(x, y) \in S\}, x \in X$, and $S^{y}=\{x \in X$ : $(x, y) \in S\}, y \in Y$, consists of exactly $n$ elements ( $n$ finite; for infinite $n$ see [8]), then $S$ contains a matching. In particular, if each section $S_{x}, x \in X$, and $S^{y}, y \in Y$, is a doubleton then $S$ contains a matching. A natural question, whether a Borel subset $S$ of $[0,1] \times[0,1]$ with doubleton sections contains a matching which is a Borel set, was settled in the negative by M. Laczkovich [12].

Call a subset $S \subset X \times Y$ good if every complex-valued function $f$ on $S$ is of the form

$$
f(x, y)=u(x)+v(y), \quad(x, y) \in S,
$$

where $u$ and $v$ are functions on $X$ and $Y$ respectively. Such sets occur in connection with some problems in ergodic theory and have a description [3]. It turns out that some very natural questions about good Borel sets have their answers in the facts about Borel sets with doubleton sections and one

2000 Mathematics Subject Classification: Primary 60A05, 47A35; Secondary 28D05, $37 \mathrm{Axx}$.

Key words and phrases: good sets, linked components, sequentially good sets, simplicial measures. 
of the purposes of this paper is to exhibit this connection. Ergodic theory intertwines the discussion and partly the paper continues the theme of [10].

In Section 1 we give the preliminaries and some crucial examples. Some basic facts about sets with doubleton sections are discussed in Section 2 while good sets are discussed similarly in Section 3. In Section 4 some natural questions about good Borel sets are answered, nearly fully, partly using results of Section 2. In Section 5 we define sequentially good measures and show that such measures admit good Borel sets as support.

After this paper was written, K. P. S. Bhaskara Rao pointed out to us that this work is connected with some old as well as recent literature on extreme points of doubly stochastic probability measures with the same marginals. Indeed, among other things, our discussion inadvertently isolates and articulates the set-theoretic content of some of this work, especially, in the basic note of J. Lindenstrauss [13] and the papers of V. Beneš and J. Štěpán [1, 2], J. Štěpán [20] and there is some overlap with the work of K. Hestir and S. Williams [7]. We also mention papers [4] and [18] with reference to doubly stochastic matrices and the beginnings by Birkhoff and von Neumann. Section 6 discusses this connection briefly. Further, M. H. Vasavada brought to our notice some literature on the question of the density of a sum of two function algebras, especially the papers of D. Marshall and A. O'Farrell $[15,16]$ which are relevant to the theme of this paper. This is discussed in Section 6 as well.

The notion of loopfree subsets of $\mathbb{R}^{2}$ is central in these papers as it is in this paper, but it is not explicated that these sets are precisely the good sets in our sense and that they coincide with sequentially good sets (see 3.3). This observation at set-theoretic level simplifies some proofs based on measure-theoretic estimates and allows an improved Borel-set-theoretic analysis. Finally, for Borel sets with doubleton sections, apart from Theorem 4.8, we are able to complete some discussions of [16, pp. 358-361], [1, pp. 39-41] (see 6.5, 6.6).

It is a pleasure to acknowledge referee's suggestions, and discussions with K. P. S. Bhaskara Rao, which led to considerable improvement of the paper.

\section{Notation and preliminaries}

1.1. Let $X$ and $Y$ be non-empty sets and $S \subset X \times Y$. For $x \in X$ and $y \in Y$, the sets

$$
S_{x}=\{(x, y):(x, y) \in S\}, \quad S^{y}=\{(x, y):(x, y) \in S\}
$$

are called $x$ - and $y$-sections of $S$ respectively. This definition of sections differs from the usual one (used above to state König's theorem) and will be more convenient for our purpose. Let $\pi_{X}$ and $\pi_{Y}$ denote the usual projections from $X \times Y$ onto the spaces $X$ and $Y$ respectively. 
Note that $S \subset X \times Y$ contains a matching if and only if there exist one-to-one maps $f: \pi_{X}(S) \rightarrow \pi_{Y}(S)$ and $g: \pi_{Y}(S) \rightarrow \pi_{X}(S)$ whose graphs are contained in $S$. The "only if" part of this statement is clear. For the "if" part, the result follows from the standard proofs of the Schröder-Bernstein theorem $([19$, p. 5$])$. Let $\Pi$ be a partition of a set $S$. The equivalence relation defined by $\Pi$ will also be denoted by $\Pi$, so it is clear what is meant when we write $x \Pi x^{\prime}$ or $\Pi \subset S \times S$. A subset $C$ of $S$ which intersects each member of $\Pi$ in exactly one point is called a cross-section of $\Pi$ (or of the equivalence classes of $\Pi$ ). Suppose $S \subset X \times Y$ with $\pi_{X}(S)=X$ and $\pi_{Y}(S)=Y$. Then the collections $\left\{S_{x}: x \in X\right\}$ and $\left\{S^{y}: y \in Y\right\}$ are partitions of $S$ and any set $C \subset S$ which is simultaneously a cross-section of $\left\{S_{x}: x \in X\right\}$ and $\left\{S^{y}: y \in Y\right\}$ is a matching of $S$.

We say that $S$ has doubleton sections if all sections $S_{x}$ and $S^{y}$ are twopoint sets. We give below some examples of such sets relevant to the paper.

1.2. EXAMPLE. The circumference $S$ of the circle with center $(1 / 2,1 / 2)$ and radius $1 / 2$ together with corner points $(0,0)$ and $(1,1)$ is contained in $[0,1] \times[0,1]$ and has doubleton sections.

1.3. Example. Let $X=Y=\mathbb{Z}$. The zig-zag set

$$
S=\{(n, n-1): n \in \mathbb{Z}\} \cup\{(n, n): n \in \mathbb{Z}\} \subset \mathbb{Z} \times \mathbb{Z}
$$

has doubleton sections.

1.4. Examples. (i) ([2]) Let $0<\theta<1$. Let $L, M, N$ respectively be the line segments in $[0,1] \times[0,1]$ joining $(\theta, 0)$ to $(1,1-\theta),(0,0)$ to $(1,1)$, $(0,1-\theta)$ to $(\theta, 1)$, and let $R$ be the union of $L, M, N$. In order to ensure that each section is a doubleton we require that $L$ contains $(\theta, 0)$ but not $(1,1-\theta), M$ contains $(0,0)$ but not $(1,1)$, and $N$ contains $(0,1-\theta)$ but not $(\theta, 1)$. Thus $S=R \backslash\{(\theta, 1),(1,1-\theta),(1,1)\} \subset[0,1) \times[0,1)$ is a set with doubleton sections. (See Figure 1.)

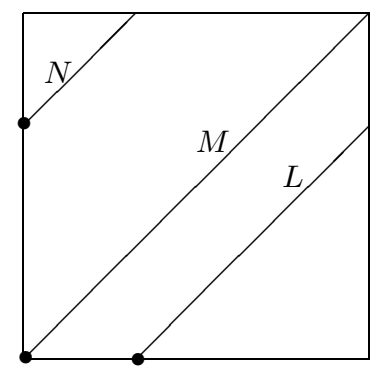

Fig. 1

(ii) More generally one can replace $N \cup L$ in the above example by the graph $G$ of an invertible (Lebesgue) measure preserving uniquely ergodic 
transformation $T$ on $[0,1)$ with the property that $T x \neq x$ for any $x$. One can define a natural probability measure on $M \cup G$ whose marginals are Lebesgue.

1.5. Example ([12]). Let $0<u<1 / 2$, and let $R$ denote the boundary of the rectangle with vertices $A_{0}(1,1-u), A_{1}(1-u, 1), A_{2}(0, u)$, and $A_{3}(u, 0)$. Let $S=R \cup\{(0,0),(1,1)\}$. (See Figure 2.) Then $S \subset[0,1] \times[0,1]$ is a set with doubleton sections.

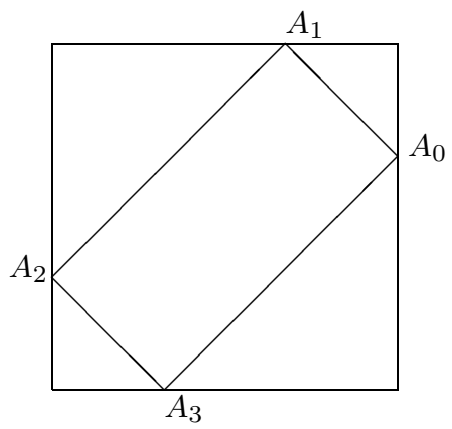

Fig. 2

1.6. The set $S$ of Example 1.3 is good (as defined in the introduction). To see this, fix any complex-valued function $f$ on $S$. If we put $u(0)=c$, where $c$ is an arbitrary constant, then $v(0)=f(0,0)-c$. Having defined $v(0)$, we see that $u(1)=f(1,0)-v(0)$ and $v(1)=f(1,1)-u(1)$. Proceeding thus we see that $u$ and $v$ are uniquely defined as soon as we fix the value of $u(0)$. We shall see later that sets $S$ of Examples 1.4 and 1.5 are good if $\theta$ and $u$ are irrational, and the one in Example 1.2 is not.

1.7. Now assume that $X$ and $Y$ are Polish spaces and $S \subset X \times Y$ Borel. Several natural questions arise. Suppose $S$ admits a matching as is the case, for example, when $S$ has doubleton sections. Is it then true that $S$ admits a Borel matching? Or assume that $S$ is good. Is it then true that in formula (1), $u$ and $v$ can be chosen to be Borel whenever $f$ is Borel? Later we shall study these problems systematically.

We close this section by giving some descriptive-set-theoretic results that will be needed. Concepts and notation used from descriptive set theory are standard and we refer the reader to [9] or [19] for this. For a metrizable space $X, \mathcal{B}_{X}$ will denote its Borel $\sigma$-algebra. A partition $\Pi$ of $X$ into Borel sets is said to be countably generated if there exist Borel sets $\left\{B_{n}: n \in \mathbb{N}\right\}$ such that for every $x, x^{\prime}$ in $X$,

$$
x \Pi x^{\prime} \Leftrightarrow \forall n\left(x \in B_{n} \Leftrightarrow x^{\prime} \in B_{n}\right) ;
$$

equivalently, $\Pi$ is induced by a Borel map $f: X \rightarrow[0,1]$. Such partitions are easily seen to be Borel, i.e., $\Pi \in \mathcal{B}_{X \times X}$. We shall need the following results on the existence of a Borel cross-section of partitions into Borel sets. 
1.8. TheOREM (Lusin, [19, p. 205]). Let $X, Y$ be standard Borel spaces and let $B \subset X \times Y$ be a Borel set. Suppose $B_{x}$ is countable for every $x \in X$. Then $\pi_{X}(B)$ is Borel and there is a Borel map $f: \pi_{X}(B) \rightarrow Y$ whose graph is contained in $B$.

1.9. Corollary. Let $X, Y$ be standard Borel spaces and $f: X \rightarrow Y$ a countable-to-one Borel map. Then $f(X)$ is Borel.

1.10. Corollary. Let $X$ be a standard Borel space and $\Pi$ a Borel partition of $X$ with each member of the partition countable. Then $\Pi$ is countably generated if and only if $\Pi$ admits a Borel cross-section.

Proof. Suppose $\Pi$ is countably generated. Then there is a Borel map $f: X \rightarrow[0,1]$ inducing $\Pi$. The set

$$
S=\{(t, x) \in[0,1] \times X: f(x)=t\}
$$

is Borel and $S_{t}$ is countable for all $t$. By Lusin's theorem (1.8), we get a Borel map $s: f(X) \rightarrow X$ whose graph is contained in $S$. The set

$$
C=\{x \in X: s(f(x))=x\}
$$

is a Borel cross-section of $\Pi$. Conversely, suppose $\Pi$ admits a Borel cross section, say $B$. Then $f$ defined by

$$
f(x)=b \Leftrightarrow x \Pi b, \quad b \in B
$$

is Borel measurable since the graph of $f$ is $\pi_{Y}^{-1}(B) \cap \Pi$, which is a Borel set. (A function is Borel measurable if and only if its graph is.) Clearly $\Pi$ is induced by $f$, hence countably generated. (Note that this part of the proof does not require the partition elements to be countable.)

1.11. TheOrEm (Novikov, [19, p. 204]). Let $\Pi$ be a Borel partition of a standard Borel space $X$ with each member of $\Pi$ compact. Then $\Pi$ admits a Borel cross-section, hence is countably generated. In particular, if each member of $\Pi$ is finite then $\Pi$ admits a Borel cross-section.

1.12. Theorem. Let $X, Y$ be Polish spaces and $S \subset X \times Y$ a Borel set with sections $S_{x}$ countable. Then the set

$$
U=\left\{x \in X: S_{x} \text { is a singleton }\right\}
$$

is Borel.

Proof. Write $S=\bigcup_{n} G_{n}$, where $G_{n}$ 's are pairwise disjoint and graphs of functions from Borel subsets of $X$ to $Y$ ([19, p. 210]). It is easily checked that

$$
U=\bigcup_{n}\left[\pi_{X}\left(G_{n}\right) \backslash \bigcup_{m \neq n} \pi_{X}\left(G_{m}\right)\right] .
$$

This completes the proof. 


\section{Sets with doubleton sections}

2.1. Let $X$ and $Y$ be Polish spaces and $S \subset X \times Y$ be a Borel set with doubleton sections. For each $(x, y) \in S$ there exists a unique $\left(x, y^{\prime}\right) \in S$ with $y \neq y^{\prime}$ and also there exists a unique $\left(x^{\prime}, y\right) \in S$ with $x \neq x^{\prime}$. For such an $S$ the maps $v:(x, y) \mapsto\left(x, y^{\prime}\right)$ and $h:(x, y) \mapsto\left(x^{\prime}, y\right)$ are Borel measurable bijections on $S$ with $h^{2}=v^{2}=e$, where $e$ denotes the identity map on $S$.

Let $\mathbb{G}$ be the (non-abelian) group generated by $v$ and $h$ and let $\mathbb{G}_{0}$ be the cyclic subgroup of $\mathbb{G}$ generated by $h v$. Label the members of $\mathbb{G}$ as

$$
\begin{aligned}
\ldots, g_{-2 n}=(v h)^{n}, g_{-2 n+1}=h(v h)^{n-1}, \ldots, g_{-1}=h, g_{0}=e, \\
g_{1}=v, g_{2}=h v, \ldots, g_{2 n}=(h v)^{n}, g_{2 n+1}=v(h v)^{n}, \ldots
\end{aligned}
$$

The subgroup $\mathbb{G}_{0}$ is the set of elements of this group with even subscripts. It is an abelian normal subgroup of $\mathbb{G}$ and admits only two cosets, namely, $\mathbb{G}_{0}$ and

$$
v \mathbb{G}_{0}=\mathbb{G}_{0} v=h \mathbb{G}_{0}=\mathbb{G}_{0} h=\left\{g_{2 n+1}: n \in \mathbb{Z}\right\} .
$$

For any $(x, y) \in S$, the $\mathbb{G}$-orbit $\mathbb{G}(x, y)$ of $(x, y)$ is a union of two $\mathbb{G}_{0}$-orbits:

$$
\mathbb{G}(x, y)=\mathbb{G}_{0}(x, y) \cup \mathbb{G}_{0}(v(x, y)) .
$$

If $C$ is a set which meets each $\mathbb{G}$-orbit in exactly one point, then $C \cup v C$ is a set which meets each $\mathbb{G}_{0}$-orbit in exactly one point. Thus, if the orbits of $\mathbb{G}$ in $S$ admit a Borel cross-section, say $C$, then the orbits of $\mathbb{G}_{0}$ in $S$ admit a Borel-cross section $C \cup v C$. On the other hand, if the orbits of $\mathbb{G}_{0}$ in $S$ admit a Borel cross-section, say $C$, then $C$ intersects each $\mathbb{G}$-orbit in exactly two points. By 1.11, this partition of $C$ admits a Borel cross-section, say $D$. Then $D$ is a Borel cross-section of $\mathbb{G}$-orbits in $S$. Thus the partition of $S$ into $\mathbb{G}$-orbits admits a Borel cross-section if and only if the partition of $S$ into $\mathbb{G}_{0}$-orbits does.

2.2. If $M$ is a matching (not necessarily Borel), then clearly

$$
M \cap h M=M \cap v M=\emptyset, \quad \text { while } \quad M \cup h M=M \cup v M=S .
$$

Any $M$ satisfying these conditions is a matching. This is equivalent to saying that a set $M$ is a matching for $S$ if and only if $M$ is $\mathbb{G}_{0}$-invariant and

$$
M \cap h M=\emptyset, \quad M \cup h M=S .
$$

Clearly $M$ is a Borel matching if and only if $M$ is a $\mathbb{G}_{0}$-invariant Borel set satisfying (2).

If, using the axiom of choice, we select a cross-section $C$ of the $\mathbb{G}$-orbits, then $M=\bigcup_{g \in \mathbb{G}_{0}} g C$ is a $\mathbb{G}_{0}$-invariant set satisfying (2), so that $M$ is a matching for $S$. So a matching $M$ always exists, but it need not be Borel. If the partition of $S$ into $\mathbb{G}$-orbits admits a Borel cross-section $C$ then clearly $M$ is a Borel matching. In particular, if each $\mathbb{G}$-orbit is a finite set, or more generally, if the partition into $\mathbb{G}$-orbits is countably generated, then we know 
from 1.10 that the $\mathbb{G}$-orbits admit a Borel cross-section, so in this case $S$ admits a Borel matching.

2.3. The condition that the partition of $S$ into $\mathbb{G}$-orbits admits a Borel cross-section, or equivalently, that the partition is countably generated, is not necessary for the existence of a Borel matching. To see this consider the set $S$ of Example 1.4 with $\theta$ irrational. The $\mathbb{G}$-orbits of points in $S$ are countable and they induce a partition on $M$ consisting of the orbits of the map

$$
\Theta:(x, x) \mapsto(x+\theta(\bmod 1), x+\theta(\bmod 1)) .
$$

Since $\theta$ is irrational, this is the Vitali partition of $M$ and so it does not admit a Borel cross-section $([19,3.4 .18])$, hence the partition of $S$ into $\mathbb{G}$ orbits does not admit a Borel cross-section. However, the diagonal $M$ is a Borel matching for $S$.

2.4. A probability measure $m$ on $S$ is called $\mathbb{G}$-quasi-invariant if $m(A)$ $=0$ implies that $m(g A)=0$ for all $g \in \mathbb{G}$. Further, we say that $\mathbb{G}$ acts ergodically with respect to $m$ (or $m$ is ergodic under $\mathbb{G}$ ) if for any $\mathbb{G}$-invariant Borel set $A$, either $m(A)=0$ or $m(S \backslash A)=0$. If $S$ admits a Borel matching, say $M$, then, since $(2)$ is satisfied, for any $\mathbb{G}$-quasi-invariant measure $m$ we have $m(M)>0, m(S \backslash M)>0$, and since $M$ is $\mathbb{G}_{0}$-invariant, $m$ cannot be $\mathbb{G}_{0}$-ergodic. We have proved: if $S$ admits a Borel matching then no $\mathbb{G}$-quasiinvariant probability measure can be ergodic under the $\mathbb{G}_{0}$-action.

We do not know if the converse of this holds. However this allows us to exhibit an example, due to Laczkovich [12], of a Borel set $S$ with doubleton sections which does not admit a Borel matching. Indeed, the set $S$ of 1.5 with $u$ irrational does not admit a Borel matching: the measure $\mu$ which agrees with the normalized linear measure on $R$ and gives zero mass to singletons $(0,0),(1,1)$ is ergodic under the action of $\mathbb{G}_{0}$, since, as proved in [12], this action is conjugate to the action $z \mapsto e^{2 \pi i u} z$ on the circle group.

2.5. Although the set $S$ above does not admit a Borel matching, one can use an argument as in Rokhlin's lemma to show that given $\varepsilon>0$, there exists a Borel set $M \subset S$ such that $M \cap h M=M \cap v M=\emptyset$ and $\mu(M \cup h M)$, $\mu(M \cup v M)>1-\varepsilon$, i.e., one can obtain an approximate Borel matching of size as large as one wishes.

2.6. We note that in Examples 1.4(i), (ii) the $\mathbb{G}$-action is uniquely ergodic but the $\mathbb{G}_{0}$-action is not uniquely ergodic, there being two ergodic components (with respect to $\mathbb{G}_{0}$ ), while in Example 1.5 the $\mathbb{G}_{0}$-action is uniquely ergodic. This phenomenon holds in general. More precisely, if $S$ is a good Borel set in the unit square with doubleton sections, and if the $\mathbb{G}$-action is uniquely ergodic and measure preserving with respect to an atomfree probability measure $m$, then either the $\mathbb{G}_{0}$-action admits exactly 
two ergodic components or the $\mathbb{G}_{0}$-action is uniquely ergodic. Further it is easy to show that in the first case the $\mathbb{G}_{0}$-action is conjugate to the $\mathbb{G}_{0}$-action on the set $S$ described in 1.4(ii), for a suitable $T$. Thus, when the $\mathbb{G}_{0}$-action admits two ergodic components we have a complete description of the $\mathbb{G}$ - as well as $\mathbb{G}_{0}$-actions. We do not know a similar description when the $\mathbb{G}_{0}$-action is uniquely ergodic.

2.7. REMARK. Let $n \geq 2$ be an integer. Divide the rectangle $[0, n] \times[0, n]$ into $n^{2}$ rectangles of equal size. Fix an irrational $u, 0<u<1 / 2$. In each of these rectangles construct a rectangle $R$ as described in 1.5. Call this set $T$. (Figure 3 shows $T$ for $n=2$.) Remove from $T$ the linked component (see the next section for the definition) of each of the corners (e.g., $A_{0}, A_{1}, \ldots, A_{15}$ in Fig. 3) of the rectangles. Call the set so obtained $S$. Set $X=\pi_{[0, n]}(S)$. Then $X$ is cocountable in $[0, n]$ and $S \subset X \times X$ is a Borel set whose sections $S_{x}, x \in X$, and $S^{y}, y \in X$, are of cardinality $2 n$. So $S$ admits a matching. But it does not admit a Borel matching. To see this, one first notes that if $S$ has a Borel matching, so does $T$. Finally one constructs a Borel matching of $T \cap([0,1] \times[0,1])$ from that of $T$. This is not possible as has been observed earlier. It is an open question whether a similar set exists for odd integers. In particular it is not known whether there exists a Borel set $S \subset[0,1] \times[0,1]$, not admitting a Borel matching, and such that the sections $S_{x}, S^{y}$ are of cardinality 3 .

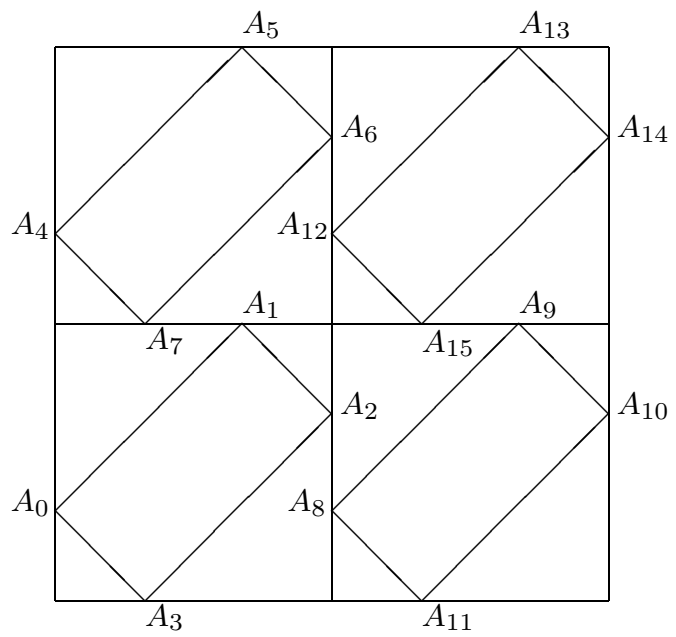

Fig. 3

\section{Good sets}

3.1. Definition. Let $S \subset X \times Y$. Two arbitrary points $(x, y),(z, w)$ in $S \subseteq X \times Y$ ( $S$ is not necessarily good) are said to be linked (and we write $(x, y) L(z, w))$ if there exists a finite sequence of points $\left(x_{1}, y_{1}\right),\left(x_{2}, y_{2}\right), \ldots$, $\left(x_{n}, y_{n}\right)$ in $S$ (called a link of length $n$ joining $(x, y)$ to $(z, w)$ ) such that 
(i) $\left(x_{1}, y_{1}\right)=(x, y),\left(x_{n}, y_{n}\right)=(z, w)$;

(ii) for any $1 \leq i \leq n-1$ exactly one of the following equalities holds:

$$
x_{i}=x_{i+1}, \quad y_{i}=y_{i+1}
$$

(iii) for any $i, 1 \leq i \leq n-2$, it is not possible to have $x_{i}=x_{i+1}=x_{i+2}$ or $y_{i}=y_{i+1}=y_{i+2}$.

The relation $L$ is an equivalence relation, as can be verified, and has appeared earlier $[16,8]$. An equivalence class of $L$ is called a linked component of $S$. If $(x, y) \in S$, then the equivalence class to which $(x, y)$ belongs is called the linked component of $(x, y)$. Two points $(x, y),(z, w) \in S$ are said to be uniquely linked if there is a unique link joining $(x, y)$ to $(z, w)$. A linked component of $S \subseteq X \times Y$ is said to be uniquely linked if any two points in it are uniquely linked. If $\left(x_{0}, y_{0}\right), \ldots,\left(x_{n}, y_{n}\right)$ is the unique link joining $\left(x_{0}, y_{0}\right)$ to $\left(x_{n}, y_{n}\right)$, then we say that $\left(x_{n}, y_{n}\right)$ is at a distance $n$ from $\left(x_{0}, y_{0}\right)$. By a trivial link joining $(x, y)$ to itself we mean the link consisting of the singleton $\{(x, y)\}$. A non-trivial link joining $(x, y)$ to itself is called a loop. It is easy to see that a linked component is uniquely linked if and only if it has no loops. If a set $S$ admits a loop then it contains a loop of smallest length which is necessarily odd.

3.2. TheOrem. A subset $S \subset X \times Y$ is good if and only if each of its linked components is uniquely linked.

Proof. If linked components are not uniquely linked then $S$ admits a loop $\left(x_{0}, y_{0}\right),\left(x_{1}, y_{1}\right), \ldots,\left(x_{2 n}, y_{2 n}\right)=\left(x_{0}, y_{0}\right)$. It is easy to see that if $f: S \rightarrow \mathbb{C}$ can be written in the form $u(x)+v(y)$ then

$$
f\left(x_{0}, y_{0}\right)-f\left(x_{1}, y_{1}\right)+\cdots+f\left(x_{2 n-1}, y_{2 n-1}\right)=0 .
$$

Since not every function $f: S \rightarrow C$ satisfies this, $S$ is not good.

In order to prove the "if" part we may assume that $S$ consists of only one uniquely linked component. Let $f: S \rightarrow \mathbb{C}$ be arbitrary. Fix $\left(x_{0}, y_{0}\right) \in S$, and let $\left(x_{0}, y_{0}\right),\left(x_{1}, y_{1}\right), \ldots,\left(x_{n}, y_{n}\right)=(x, y)$ be the link joining $\left(x_{0}, y_{0}\right)$ to a point $(x, y) \in S$. Starting with $u\left(x_{0}\right)=c$ it is easy to see how to define $u\left(x_{n}\right), v\left(x_{n}\right)$ (see 1.6) so that $u(x)+v(y)=f(x, y)$. Since $S$ has no loops, $u(x)$ and $v(y)$ are well defined for all $(x, y) \in S$ and they satisfy $f(x, y)=u(x)+v(y)$.

REMARK. For a function $f$ on a good set $S$ one can write down formulas for $u$ and $v$ which satisfy (1). Let $C$ be a cross-section of the linked components of $S$ and let a generic point $(x, y) \in S$ be linked to a point $\left(x_{0}, y_{0}\right) \in C$ by the $\operatorname{link}\left(x_{0}, y_{0}\right),\left(x_{1}, y_{1}\right), \ldots,\left(x_{n}, y_{n}\right)(=(x, y))$. Assume that this link begins by moving in the vertical direction, so that $x_{0}=x_{1}$. 
We define, with $n=2 k$ or $2 k+1$,

$$
u(x)=u\left(x_{n}\right)=\sum_{j=0}^{2 k}(-1)^{j} f\left(x_{j}, y_{j}\right)-v\left(y_{0}\right), \quad v(y)=f(x, y)-u(x),
$$

and note that (1) holds. One can write down similar formulas in case the link joining $\left(x_{0}, y_{0}\right)$ to $\left(x_{n}, y_{n}\right)$ begins with the first step in the horizontal direction, i.e., if $x_{0} \neq x_{1}$. Outside $X \backslash \pi_{X} S$ and $Y \backslash \pi_{Y} S$, we can define $u, v$ arbitrarily.

3.3. Call a set $S \subset X \times Y$ sequentially good if every complex-valued function $f$ on $S$ is the pointwise limit of a sequence of functions of the form $u_{n}(x)+v_{n}(y),(x, y) \in S, n=1,2, \ldots$, with $u_{n}, v_{n}$ defined on $X$ and $Y$ respectively. From the last theorem it is easily observed that a set is good $\Leftrightarrow$ it is sequentially good $\Leftrightarrow$ each of its finite subsets is good ([10]). Further, if $S$ has doubleton sections, then $S$ is good whenever each $\mathbb{G}$-orbit is infinite.

\section{Good Borel sets}

4.1. Assume now that $X$ and $Y$ are Polish spaces and that $S \subset X \times Y$ is a Borel set such that every Borel function $f$ on $S$ is of the form (1). Then $S$ is good, for otherwise $S$ will admit a loop $L$ (a finite set, hence a Borel set) and we can define a Borel function $f$ on $S$ which cannot be written in the form $f(x, y)=u(x)+v(y)$ on $L$, hence on $S$.

4.2. The following descriptive questions naturally arise concerning a good Borel set $S$ and its partition $\Pi$ into linked components.

A) Is $\Pi$ countably generated?

B) If $\Pi$ is countably generated, is the quotient $\sigma$-algebra on the linked components standard Borel?

C) If $f$ is a Borel function on $S$, can one choose functions $u$ and $v$ on $X$ and $Y$ respectively which satisfy (1) to be Borel measurable?

D) If $S$ is a good set then, as proved in [7,3], we can write $S$ as a union of two graphs $G$ and $H$ of functions defined on subsets of $X$ and $Y$ respectively. Can one choose the graphs $G$ and $H$ to be Borel sets if we know in addition that $S$ is a Borel set?

4.3. Without the knowledge about Borel sets with doubleton sections one would be inclined to conjecture that questions A), C) and D) have affirmative answers, but all the questions above have negative answers.

(i) For question A) we look at Example 1.4 with $\theta$ irrational. The linked components of $S$ are uniquely linked and they form orbits of the action of $\mathbb{G}$ on $S$. The partition induced by the linked components on the diagonal $M=\{(x, x): 0 \leq x<1\}$ is the Vitali partition, which is not countably generated. So $\Pi$ is not countably generated. 
(ii) For question B), let $S$ be the graph of a Borel function from $X$ to $Y$ whose image is analytic but not Borel. Then the quotient Borel structure on the partition $\Pi$ into linked components of $S$ is isomorphic to the Borel structure on $\pi_{Y}(S)$. So it is countably generated, but is not standard Borel. It would be interesting to construct such an example with $\pi_{X}(S), \pi_{Y}(S)$ Borel.

(iii) For question C) we revert to the set $S$ of Example 1.4 with $\theta$ irrational and take $f=I_{M}$, where $I_{M}$ is the indicator function of $M$. Assume that $u$ and $v$ are functions on $[0,1)$ satisfying (1). We show that $u$, hence $v$, cannot be Borel measurable. Fix $\left(x_{0}, y_{0}\right)\left(=\left(x_{0}, x_{0}\right)\right) \in M$ and let $\left(x_{k}, y_{k}\right)=g_{k}\left(x_{0}, y_{0}\right)$, where $g_{k}$ is as defined in 2.1. Note that $f\left(x_{k}, y_{k}\right)=0$ or 1 , depending on whether $k$ is odd or even. Note that for $k \geq 0, x_{2 k}=x_{2 k+1}$ and

$$
u\left(x_{2 k}\right)=\sum_{j=0}^{2 k}(-1)^{j} 1_{M}\left(x_{j}, y_{j}\right)-v\left(y_{0}\right)=k+1-v\left(y_{0}\right) .
$$

For $k<0, x_{2 k}=x_{2 k+1}$, and

$$
u\left(x_{2 k+1}\right)=u\left(x_{2 k}\right)=\sum_{j=1}^{-2 k-1}(-1)^{j+1} 1_{M}\left(x_{-j}, y_{-j}\right)=k+1-v\left(y_{0}\right) .
$$

The set $\left\{x_{2 k}: k \in \mathbb{Z}\right\}$ is the orbit of $x_{0}$ under the map

$$
\Theta: x \mapsto x+\theta(\bmod 1) .
$$

The map $k \mapsto u\left(x_{2 k}\right)$ is strictly increasing and has range $\mathbb{Z}-v\left(y_{0}\right)$. The inverse image $u^{-1}([0,1))$ intersects each $\Theta$-orbit in exactly one point, whence it is a cross-section of the Vitali partition, consequently not a Borel set. So $u$ cannot be Borel measurable.

The proof in fact shows that if $S$ is a good set with doubleton sections, admitting a Borel matching $M$, and such that the partition into linked components is not countably generated, then $u$ and $v$ for $I_{M}$ cannot be chosen to be Borel functions.

(iv) To answer question D) in the negative, let $S$ be the Laczkovich set (see 1.5) with $u$ irrational. Since $u$ is irrational, each $\mathbb{G}$-orbit (hence each linked component of $S$ ) is of infinite cardinality, so $S \subset[0,1] \times[0,1]$ is a good Borel set. Suppose $S=G \cup H$, a union of graphs of Borel functions on Borel subsets of $X$ and $Y$ respectively. Note that $\pi_{X}(H)=X$ and $\pi_{Y}(G)=Y$. Write

$$
\begin{aligned}
& G_{1}=\{(x, y) \in G: h(x, y) \in G\}, \\
& G_{2}=\left\{(x, y) \in G_{1}: \pi_{X}(x, y)<\pi_{X} h(x, y)\right\}, \quad G_{3}=G \backslash h G_{2} .
\end{aligned}
$$

Note that $\pi_{Y}\left(G_{3}\right)=\pi_{Y}(G)=Y$, and $G_{3}$ is the graph of a one-to-one function on $Y$. Thus, there exists a one-to-one Borel map $f: Y \rightarrow X$ whose 
graph is contained in $S$. By symmetry, there also exists a one-to-one Borel map $g: X \rightarrow Y$ whose graph is contained in $S$. From standard proofs of the Schröder-Bernstein theorem ([19, p. 5]), it follows that $S$ admits a Borel matching and this is a contradiction.

Remark. That it may not always be possible to choose these graphs to be Borel measurable follows (among other things) from an example of V. Losert [14] (see [1, 20]). However the above example is simpler.

4.4. We will now show that if the linked components of a good Borel set $S$ admit a Borel cross-section, then the questions A), B), C) and D) above have positive answers (see also [7]). However, before proving this, it is necessary to give the partition $\Pi$ of linked and uniquely linked components a careful look.

Let $S \subset X \times Y$ be a Borel set (not necessarily good). Let

$$
S^{n}=\left\{\left(\left(x_{1}, y_{1}\right), \ldots,\left(x_{n}, y_{n}\right)\right):\left(x_{i}, y_{i}\right) \in S, 1 \leq i \leq n\right\} .
$$

Define $\pi_{i}: S^{n} \rightarrow S, 1 \leq i \leq n$, by

$$
\pi_{i}\left(\left(x_{1}, y_{1}\right), \ldots,\left(x_{n}, y_{n}\right)\right)=\left(x_{i}, y_{i}\right) .
$$

An element

$$
s=\left(\left(x_{1}, y_{1}\right), \ldots,\left(x_{n}, y_{n}\right)\right) \in S^{n}
$$

is a link joining $\left(x_{1}, y_{1}\right)$ to $\left(x_{n}, y_{n}\right)$ with $x_{1}=x_{2}$ if and only if finitely many conditions of the form

$$
\begin{array}{ll}
\pi_{X} \pi_{1}(s)=\pi_{X} \pi_{2}(s), & \pi_{Y} \pi_{1}(s) \neq \pi_{Y} \pi_{2}(s), \\
\pi_{X} \pi_{2}(s) \neq \pi_{X} \pi_{3}(s), & \pi_{Y} \pi_{2}(s)=\pi_{Y} \pi_{3}(s),
\end{array}
$$

are satisfied. Similarly, we can describe the set of all links $s$ of length $n$ with $y_{1}=y_{2}$.

Thus the set $Q^{n}$ of points $\left(\left(x_{1}, y_{1}\right), \ldots,\left(x_{n}, y_{n}\right)\right)$ in $S^{n}$ which form a link in $S$ is a Borel subset of $S^{n}$. The set $Q_{n}$ of all pairs $\left(\left(x_{1}, y_{1}\right),\left(x_{n}, y_{n}\right)\right)$ obtained by projecting $Q^{n}$ is therefore an analytic set. It consists of all pairs $((x, y),(z, w))$ such that $(x, y)$ is linked to $(z, w)$ by a path of length $n$. The set $\Pi=\bigcup_{n=1}^{\infty} Q_{n}$, which is the set of all pairs $((x, y),(z, w))$ with $(x, y) L(z, w)$, is therefore an analytic set. The horizontal and vertical sections of $\Pi$ are the linked components of $S$ and they are also analytic sets.

The following example, due to the referee, shows that in general the partition into linked components need not be a Borel equivalence relation. Let $X=Y=\mathbb{R}$, and let $B \subset[0,1] \times[0,1]$ be a Borel set such that $\pi_{X}(B)=A$ is not Borel. We may assume that $(0,0) \in B$. We put $C=\{0\} \times[0,1]$, $D=\{(x,-x): x \in[0,1]\}$, and $S=B \cup C \cup D$. The set $S$ is Borel. Let $E$ be the linked component containing $(0,0)$. We claim that $E \cap D=\{(x,-x)$ : $x \in A\}$. Indeed, if $(x,-x) \in E \cap D$ then any link $\left(x_{1}, x_{1}\right), \ldots,\left(x_{n}, y_{n}\right)$ joining $(x,-x)$ to $(0,0)$ must have $\left(x_{2}, y_{2}\right) \in B$, so that $x_{2}=x_{1}=x \in \pi_{X}(B)=A$. 
On the other hand, if $x \in A$ then there is a $y$ such that $(x, y) \in B$ and then $(0,0),(0, y),(x, y),(x,-x)$ is a link joining $(0,0)$ to $(x,-x)$. Since $A$ is not Borel, it is clear that $E$ is not Borel either, and then the same is true for $\Pi$. (This answers a question posed in [10, pp. 389-390].)

Now, assume that $S$ is good, or equivalently, uniquely linked. Then the above projection from $Q^{n}$ to $Q_{n}$ is in fact one-to-one, so that by $1.9, Q_{n}$ is Borel. Hence, the equivalence relation $\Pi$ and all the linked components of $S$ are Borel. We have proved: if the linked components of $S$ are uniquely linked, then the equivalence relation $L$ (which is the same as $\Pi$ ) is a Borel equivalence relation.

Let $S$ be a good Borel set and assume further that the partition $\Pi$ of $S$ admits a Borel cross-section, say $C$. The function $f: S \rightarrow C$ given by $f(x)=c$ whenever $x \Pi c, c \in C$, is Borel (see 1.10), it induces the partition $\Pi$ and the quotient $\sigma$-algebra on $\Pi$ is isomorphic to the $\sigma$-algebra on $C$, by a result of Blackwell and Mackey $([19,4.5 .10])$. Thus questions A) and B) have affirmative answers if $\Pi$ admits a Borel cross-section.

4.5. We continue with the assumption that the linked components of the Borel measurable $S$ are uniquely linked and that they admit a Borel cross-section $C$. The maps $\pi_{X}$ and $\pi_{Y}$ are one-to-one on $C$. The set

$$
R_{n}=\left\{\left(\left(x_{1}, y_{1}\right), \ldots,\left(x_{n}, y_{n}\right)\right) \in Q^{n}:\left(x_{1}, y_{1}\right) \in C\right\}=\pi_{1}^{-1}(C) \cap Q^{n}
$$

is a Borel set. Since linked components are uniquely linked, the projection $\pi_{n}$ is one-to-one on $R_{n}$. Therefore the set $C_{n}=\pi_{n} R_{n}$ is Borel and consists of points $(z, w) \in S$ which are joined to some point in $C$ (which is unique) by a path of length $n$. Clearly $S=\bigcup_{n=1}^{\infty} C_{n}$, the union being disjoint, and $C_{1}=C$.

Given $\left(x_{n}, y_{n}\right) \in C_{n}$, there is a unique $\left(x_{1}, y_{1}\right) \in C$ in the linked component of $\left(x_{n}, y_{n}\right)$, so the link $\left(\left(x_{1}, y_{1}\right), \ldots,\left(x_{n}, y_{n}\right)\right)$ is uniquely determined. Hence the map $B_{n-1}:\left(x_{n}, y_{n}\right) \mapsto\left(x_{n-1}, y_{n-1}\right)$ is well defined and Borel from $C_{n}$ into $C_{n-1}$. Indeed $B_{n-1}=\left.\pi_{n-1} \circ \pi_{n}^{-1}\right|_{C_{n}}$. (Here $\pi_{n}$ is restricted to $R_{n}$ and $\pi_{n}^{-1}$ is considered from $C_{n}$ to $R_{n}$.) More generally, the maps $B_{n-i}: C_{n} \mapsto C_{n-i}$ given by $\left(x_{n}, y_{n}\right) \mapsto\left(x_{n-i}, y_{n-i}\right), 1 \leq i \leq n$, are Borel measurable.

4.6. Now let $f$ be a complex-valued Borel function on $S$. Let $v$ be an arbitrary complex-valued Borel function on $\pi_{Y} C$. Then the formulas for $u$ and $v$ given in 3.2 can be expressed in terms of $f,\left.v\right|_{\pi_{X} C}$ and the maps $B_{k}$, and so they are Borel measurable. We have proved $[7,10]$ :

THEOREM. If $S$ is a good Borel set and the linked components admit a Borel cross-section then we can write every complex-valued Borel function $f$ on $S$ in the form (1) with $u, v$ Borel measurable.

Similarly one can prove: 
4.7. TheOREM. If $S$ is a good Borel set and the partition into linked components admits a Borel cross-section, then $S$ can be expressed as a union of two Borel measurable graphs $G$ and $H, G$ being the graph of a Borel function defined on a Borel subset of $X$ and $H$ being the graph of a Borel function defined on a Borel subset of $Y$.

We do not know whether the conclusion of Theorem 4.6 is valid under the weaker hypothesis that the linked components are countably generated. If the linked components are the orbits of a continuous action of a Polish group then the answer is affirmative in the light of a theorem of Burgess $([19,5.6])$, according to which such group actions admit a Borel cross-section whenever the partition into orbits is countably generated. On the other hand, it seems plausible that the conclusion of Theorem 4.6 fails if the linked components are not countably generated. We show this for Borel sets with doubleton sections. In other words we prove:

4.8. TheOrem. Let $S \subset X \times Y$ be a good Borel set with doubleton sections such that its partition $\Pi$ into $\mathbb{G}$-orbits is not countably generated. Then there is a Borel set $M \subset S$ such that $I_{M}$ cannot be expressed as $u(x)+$ $v(y),(x, y) \in S$, with $u, v$ Borel measurable. In particular, the Laczkovich set admits such a set $M$.

Proof. Recall that $\mathbb{G}$ is the countable group generated by $v$ and $h$ and we have enumerated $\mathbb{G}$ in Section 2.1. Also $\mathbb{G}_{0}$ denotes the group generated by $h v$. Since $S$ is good there are no loops, and since the $\mathbb{G}$-orbits are precisely the linked components, we see that the $\mathbb{G}$-action is free in the sense that $g(x, y)=(x, y)$ only if $g=e$. Now, adapting the method of Glimm and Effros $([17,21])$, one can construct a Borel set $D \subset S$ such that

(i) $\Pi \cap D$ is not countably generated,

(ii) for any $(x, y) \in D, g_{2 k}(x, y) \in D$ for infinitely many positive and infinitely many negative $k$,

(iii) for any $(x, y) \in D, g_{2 k+1}(x, y) \notin D$ for any $k$. (See Theorem 4.10.)

The Borel set $M=\bigcup_{g \in \mathbb{G}} g D$ has doubleton sections and admits a Borel matching given by $\bigcup_{g \in \mathbb{G}_{0}} g D$. To see this, it is enough to verify that

$$
M \cup h M=M \cup v M=\bigcup_{g \in \mathbb{G}} g D, \quad M \cap h M=M \cap v M=\emptyset,
$$

which is easily done. Moreover, by property (i) of $D$, the partition of $\bigcup_{g \in \mathbb{G}} g D$ into linked components is not countably generated. So, as in the solution of question $\mathrm{C}$ ) above, the function $I_{M}$ cannot be expressed as $u+v$ with $u, v$ Borel. This proves the theorem.

REMARK. Since the linked components of a Borel set with doubleton sections are countable, by 1.10, they admit a Borel cross-section whenever 
they are countably generated. In such a case, by $4.6, u, v$ can be chosen to be Borel measurable for Borel measurable $f$. Thus, for Borel sets with doubleton sections we can choose $u$ and $v$ to be Borel measurable for Borel $f$ if and only if the linked components are countably generated.

4.9. It remains to show that under the hypothesis of Theorem 4.8 a set $D$ with the required properties can be constructed. By a theorem of Ramsay and Mackey ([21], [17, Chapter 8], [19, Section 3.2]), we can give $S$ a Polish topology which generates the $\sigma$-algebra $\mathcal{B}_{S}$ and for which each $g \in \mathbb{G}$ is a homeomorphism. A point $(x, y) \in S$ is called recurrent if there exist $g_{n} \in \mathbb{G}$, $n=1,2, \ldots$, such that for all $n, g_{n}(x, y) \neq(x, y)$ but $g_{n}(x, y) \rightarrow(x, y)$. A result of B. Weiss ([21], [18, Chapter 9]) states that if a countable group of homeomorphisms on a Polish space does not admit a recurrent point then the partition into $\mathbb{G}$-orbits admits a Borel cross-section. We will use this fact in the proof of the next theorem.

4.10. Theorem. Let $S \subset X \times Y$ be a good Borel set with doubleton sections such that the partition $\Pi$ into $\mathbb{G}$-orbits does not admit a Borel cross-section. Then there exists a Borel set $D \subset S$ such that for all $(x, y) \in$ $D, g_{2 k}(x, y) \in D$ for infinitely many positive $k$ and for infinitely many negative $k$, while $g_{2 k+1}(x, y) \notin D$ for all $k$. Further $\Pi \cap D$ is not countably generated.

Proof. We give $S$ a Polish topology as in 4.9. Since the $\mathbb{G}$-orbits do not admit a Borel cross-section, we know that the $\mathbb{G}_{0}$-orbits do not admit a Borel cross-section. Hence there is an $\left(x_{0}, y_{0}\right) \in S$ which is a recurrent point for the $\mathbb{G}_{0}$-action. For a set $A \subset S$, and $(x, y) \in A$, we say that $(x, y)$ is of even index relative to $A$ if the smallest positive integer $n$ such that $g_{n}(x, y) \in A$ is even. Note that if every $(x, y) \in A$ is of even index then for all $(x, y) \in A$ the integers $n \in \mathbb{Z}$ such that $g_{n}(x, y) \in A$ are even.

We will construct a Cantor-like Borel set $D \subset S$ each of whose points has even index. Moreover the partition $\Pi \cap D$ will not be countably generated. We construct inductively Borel sets $C(1) \supset C(2) \supset \ldots \supset C(k) \supset \ldots$ such that their intersection, say $C$, when modified by a countable set, will be our set $D$.

Let $\left(x_{0}, y_{0}\right)$ be a recurrent point for $\tau:=h v$. Let $N(0)$ be a neighbourhood of $\left(x_{0}, y_{0}\right)$ such that (a) the closures of $N(0), h N(0), v N(0), h v N(0)$, $v h v N(0)$ are pairwise disjoint, (b) the diameters of all these sets are less than 1 . Write $N(1)=h v N(0)$ and let $n(0)=1$. Let $n(1)>1$ be the first integer such that $\tau^{n(1)}\left(x_{0}, y_{0}\right) \in N(0)$. Since $\left(x_{0}, y_{0}\right)$ is a recurrent point, such an integer exists. Let $N(0,0)$ be a neighbourhood of $\left(x_{0}, y_{0}\right)$ such that (a) the closures of $N(0,0)$ and $\tau^{n(1)} N(0,0)$ are contained in $N(0)$, (b) the closures of the open sets $\left\{g_{i}(N(0,0)): 0 \leq i \leq 4 n(1)\right\}$ are pairwise disjoint, (c) the diameters of $\left\{g_{i}(N(0,0)): 1 \leq i \leq 4 n(1)+2\right\}$ are less than $1 / 2$. 
Define

$$
\begin{aligned}
& N(0,1)=\tau^{n(1)} N(0,0), \quad N(1,0)=h v N(0,0), \\
& N(1,1)=h v \tau^{n(1)} N(0,0)=\tau^{n(1)+1} N(0,0) .
\end{aligned}
$$

Note that the closures of $N(1,0), N(1,1)$ are contained in $N(1)$.

Write

$$
\begin{aligned}
& C(1)=N(0) \cup N(1), \\
& C(2)=N(0,0) \cup N(0,1) \cup N(1,0) \cup N(1,1) \subset C(1) .
\end{aligned}
$$

Now $v N(0,0)$ is disjoint from $C(1)$, hence from $C(2)$, and since $h v N(0,0)=$ $N(1,0)$, the index of any point in $N(0,0)$ with respect to $C(2)$ is 2 , which is even. Since

$$
v h v N(0,0), h v h v N(0,0), \ldots, v(h v)^{(n(1)-1)} N(0,0)
$$

are disjoint from each $N(i, j)$, while

$$
(h v)^{n(1)-1} h v N(0,0)=(h v)^{n(1)} N(0,0)=N(0,1),
$$

we see that the index of any point in $N(1,0)$ with respect to $C(2)$ is $2 n(1)$, which is even. Since $v N(0,1)$ is disjoint from $C(1)$, hence from $C(2)$, while $h v N(0,1)=N(1,1)$, we see that the index of any point in $N(0,1)$ with respect to $C(2)$ is 2 , which is even. Thus the index of any point in $N(0,0) \cup$ $N(0,1) \cup N(1,0)$ with respect to $C(2)$ is even.

Suppose for a positive integer $k \geq 1$ we have chosen integers $n(1)<\ldots<$ $n(k)$ with $n(j)>n(1)+\ldots+n(j-1)$, and suppose further that for each positive integer $p \leq k$ and each $p$-term sequence $\left(\varepsilon_{1}, \ldots, \varepsilon_{p}\right)$ of zeros and ones we have chosen open sets $N\left(\varepsilon_{1}, \ldots, \varepsilon_{p}\right)$ satisfying $\left(x_{0}, y_{0}\right) \in N\left(0, \ldots, 0_{p}\right)$ and the following six conditions:

(1) $N\left(\varepsilon_{1}, \ldots, \varepsilon_{p}\right) \cap N\left(\varepsilon_{1}^{\prime}, \ldots, \varepsilon_{p}^{\prime}\right)=\emptyset$ if $\left(\varepsilon_{1}, \ldots, \varepsilon_{p}\right) \neq\left(\varepsilon_{1}^{\prime}, \ldots, \varepsilon_{p}^{\prime}\right)$.

(2) $\operatorname{cl} N\left(\varepsilon_{1}, \ldots, \varepsilon_{p+1}\right) \subset N\left(\varepsilon_{1}, \ldots, \varepsilon_{p}\right)$, where $\mathrm{cl}$ is the closure operator.

(3) $\operatorname{diam} N\left(\varepsilon_{1}, \ldots, \varepsilon_{p}\right)<1 / p$.

(4) If $i_{1}, \ldots, i_{l}$ are the places where 1 occurs in $\left(\varepsilon_{1}, \ldots, \varepsilon_{p}\right)$, then

$$
N\left(\varepsilon_{1}, \ldots, \varepsilon_{p}\right)=\tau^{n\left(i_{1}\right)+\ldots+n\left(i_{l}\right)} N\left(0, \ldots, 0_{p}\right)
$$

where $0_{p}$ indicates that the length of the sequence of zeros is $p$.

(5) The closures of the sets $g_{i} N\left(0, \ldots, 0_{p}\right), 0 \leq i \leq 4 n(p)$, are pairwise disjoint.

(6) If $C_{p}=\bigcup N\left(\varepsilon_{1}, \ldots, \varepsilon_{p}\right)$, where the union is over all $p$-term sequences of zeros and ones, then the index of any $(x, y) \in C_{p}-N\left(1, \ldots, 1_{p}\right)$ with respect to $C_{p}$ is even.

Let $n(k+1)$ be the first positive integer such that

$$
\tau^{n(k+1)}\left(x_{0}, y_{0}\right) \in N\left(0, \ldots, 0_{k}\right)
$$


From $(5)$ and the fact that $n(k)>n(1)+\cdots+n(k-1)$, we see that

$$
n(k+1) \geq 2 n(k)>n(k)+n(k-1)+\cdots+n(1) .
$$

Let $N\left(0, \ldots, 0_{k+1}\right)$ be a neighbourhood $\left(x_{0}, y_{0}\right)$ such that the closures of $\left\{g_{i} N\left(0, \ldots, 0_{k+1}\right): 0 \leq i \leq 4 n(k+1)\right\}$ are pairwise disjoint. The size of $N\left(0, \ldots, 0_{k+1}\right)$ can be further reduced, if necessary, to ensure that conditions $(\mathrm{A})$ and $(\mathrm{B})$ given below are satisfied:

(A) $\operatorname{cl}\left(\tau^{n(k+1)} N\left(0, \ldots, 0_{k+1}\right)\right) \subset N\left(0, \ldots, 0_{k}\right)$.

(B) Define $N\left(0, \ldots, 0,1_{k+1}\right)=\tau^{n(k+1)} N\left(0, \ldots, 0_{k+1}\right)$. More generally, let $\left(\varepsilon_{1}, \ldots, \varepsilon_{k+1}\right)$ be a $(k+1)$-tuple of zeros and ones and let $j_{1}<\ldots<j_{l}$ be the places of 1 in $\left(\varepsilon_{1}, \ldots, \varepsilon_{k+1}\right)$. Define

$$
N\left(\varepsilon_{1}, \ldots, \varepsilon_{k+1}\right)=\tau^{n\left(j_{1}\right)+\cdots+n\left(j_{l}\right)} N\left(0, \ldots, 0_{k+1}\right) .
$$

We choose $N\left(0, \ldots, 0_{k+1}\right)$ so that for each $N\left(\varepsilon_{1}, \ldots, \varepsilon_{k+1}\right)$,

$$
\operatorname{diam} N\left(\varepsilon_{1}, \ldots, \varepsilon_{k+1}\right)<1 /(k+1) \text {. }
$$

Note that $n(k+1)$ and the open $\operatorname{sets} N\left(\varepsilon_{1}, \ldots, \varepsilon_{k+1}\right)$ satisfy:

(I) $n(k+1)>n(1)+\cdots+n(k)$.

(II) $\operatorname{cl} N\left(\varepsilon_{1}, \ldots, \varepsilon_{k+1}\right) \subset N\left(\varepsilon_{1}, \ldots, \varepsilon_{k}\right)$. We see this as follows: if $\varepsilon_{k+1}=0$ then

$$
\begin{aligned}
\operatorname{cl} N\left(\varepsilon_{1}, \ldots, \varepsilon_{k+1}\right) & =\operatorname{cl} \tau^{n\left(j_{1}\right)+\cdots+n\left(j_{l}\right)} N\left(0, \ldots, 0_{k+1}\right) \\
& \subset \tau^{n\left(j_{1}\right)+\cdots+n\left(j_{l}\right)} N\left(0, \ldots, 0_{k}\right)=N\left(\varepsilon_{1}, \ldots, \varepsilon_{k}\right),
\end{aligned}
$$

while if $\varepsilon_{k+1}=1$ and if $j_{1}, \ldots, j_{l}$ are the places where 1 occurs in $\left(\varepsilon_{1}, \ldots, \varepsilon_{k}\right)$, then

$$
\begin{aligned}
\operatorname{cl} N\left(\varepsilon_{1}, \ldots, \varepsilon_{k+1}\right) & =\operatorname{cl} \tau^{n\left(j_{1}\right)+\cdots+n\left(j_{l}\right)+n(k+1)} N\left(0, \ldots, 0_{k+1}\right) \\
& \subset \tau^{n\left(j_{1}\right)+\cdots+n\left(j_{l}\right)} N\left(0, \ldots, 0_{k}\right)=N\left(\varepsilon_{1}, \ldots, \varepsilon_{k}\right) .
\end{aligned}
$$

(III) $\operatorname{diam} N\left(\varepsilon_{1}, \ldots, \varepsilon_{k+1}\right)<1 /(k+1)$. Further,

$$
N\left(\varepsilon_{1}, \ldots, \varepsilon_{k+1}\right) \cap N\left(\varepsilon_{1}^{\prime}, \ldots, \varepsilon_{k+1}^{\prime}\right)=\emptyset
$$

whenever $\left(\varepsilon_{1}, \ldots, \varepsilon_{k+1}\right) \neq\left(\varepsilon_{1}^{\prime}, \ldots, \varepsilon_{k+1}^{\prime}\right)$.

(IV) If $i_{1}, \ldots, i_{l}$ are the places where 1 occurs in $\left(\varepsilon_{1}, \ldots, \varepsilon_{k+1}\right)$, then

$$
N\left(\varepsilon_{1}, \ldots, \varepsilon_{k+1}\right)=\tau^{n\left(i_{1}\right)+\cdots+n\left(i_{l}\right)} N\left(0, \ldots, 0_{k+1}\right) .
$$

(V) The closures of $g_{i} N\left(0, \ldots, 0_{k+1}\right), 0 \leq i \leq 4 n(k+1)$, are pairwise disjoint.

(VI) Let $C(k+1)=\bigcup N\left(\varepsilon_{1}, \ldots, \varepsilon_{k+1}\right)$, where the union is taken over all $(k+1)$-tuples of zeros and ones. Let $\left(\varepsilon_{1}, \ldots, \varepsilon_{k+1}\right) \neq\left(1, \ldots, 1_{k+1}\right)$ and let $(x, y) \in N\left(\varepsilon_{1}, \ldots, \varepsilon_{k+1}\right)$. If $\varepsilon_{1}=0$, then

$$
N\left(\varepsilon_{1}, \ldots, \varepsilon_{k+1}\right) \subset N\left(\varepsilon_{1}, \ldots, \varepsilon_{k}\right) \subset \ldots \subset N\left(0, \ldots, 0_{k}\right) \subset N_{0},
$$


so that $v(x, y) \notin C_{k+1}$, while

$$
h v N\left(\varepsilon_{1}, \ldots, \varepsilon_{k+1}\right)=N\left(1, \ldots, \varepsilon_{k+1}\right) \subset C_{k+1},
$$

so that $(x, y)$ has index 2 (hence even) with respect to $C_{k+1}$.

$$
\begin{aligned}
& \text { If }(x, y) \in N\left(\varepsilon_{1}, \ldots, \varepsilon_{k+1}\right) \text { and if } \\
& \qquad \varepsilon_{1}=\varepsilon_{2}=\ldots=\varepsilon_{p}=1, \quad \varepsilon_{p+1}=0, \quad 2 \leq p+1 \leq k+1,
\end{aligned}
$$

then the closures of the sets

$$
v \tau^{n(1)+\cdots+n(p)} N\left(0, \ldots, 0_{p+1}\right), \quad h v \tau^{n(1)+\cdots+n(p)} N\left(0, \ldots, 0_{p+1}\right)
$$

and so on up to the set

$$
\begin{aligned}
v \tau^{n(p+1)-1} N\left(0, \ldots, 0_{p+1}\right) & \\
& =v \tau^{n(p+1)-(n(1)+\cdots+n(p))-1} \tau^{n(1)+\cdots+n(p)} N\left(0, \ldots, 0_{p+1}\right)
\end{aligned}
$$

are disjoint from the closure of $N\left(\varepsilon_{1}^{\prime}, \ldots, \varepsilon_{p+1}^{\prime}\right)$ for all $\left(\varepsilon_{1}^{\prime}, \ldots, \varepsilon_{p+1}^{\prime}\right)$, hence disjoint from $C_{k+1}$, while

$$
\begin{aligned}
\tau^{n(p+1)-(n(1)+\cdots+n(p))} \tau^{n(1)+\cdots+n(p)} N\left(0, \ldots, 0_{p+1}\right) & \\
= & \tau^{n(p+1)} N\left(0, \ldots, 0_{p+1}\right)=N\left(0, \ldots, 0,1_{p+1}\right) .
\end{aligned}
$$

Thus

$$
\tau^{(n(p+1)-(n(1)+\cdots+n(p)))} N\left(\varepsilon_{1}, \ldots, \varepsilon_{k+1}\right)=N\left(0, \ldots, 0_{p}, 1_{p+1}, \varepsilon_{p+2}, \ldots, \varepsilon_{k+1}\right),
$$

and the above considerations show that the index of any $(x, y) \in N\left(\varepsilon_{1}, \ldots\right.$ $\ldots, \varepsilon_{k+1}$ ) (where $\left.\varepsilon_{1}=1, \ldots, \varepsilon_{p}=1, \varepsilon_{p+1}=0,2 \leq p+1 \leq k+1\right)$ with respect to $C(k+1)$ is even, being equal to $2(n(p+1)-n(1)-\cdots-n(p))$. Now let $C=\bigcap_{k=1}^{\infty} C(k)$, which, with induced topology, is homeomorphic to $\{0,1\}^{\mathbb{N}}$. Indeed if $(x, y) \in C$, then there is a unique infinite sequence $\left\{\varepsilon_{k}\right\}_{k=1}^{\infty}$ of zeros and ones such that $\{(x, y)\}=\bigcap_{k=1}^{\infty} N\left(\varepsilon_{1}, \ldots, \varepsilon_{k}\right)$, while given any sequence of $\left\{\varepsilon_{k}\right\}_{k=1}^{\infty}$ of zeros and ones the set $\bigcap_{k=1}^{\infty} N\left(\varepsilon_{1}, \ldots, \varepsilon_{k}\right)$ is a unique point of $C$ by the Cantor intersection theorem. Let $\phi$ denote the map from $\{0,1\}^{\mathbb{N}}$ to $C$ given by

$$
\phi\left(\left\{\varepsilon_{k}\right\}_{k=1}^{\infty}\right)=\bigcap_{k=1}^{\infty} N\left(\varepsilon_{1}, \ldots, \varepsilon_{k}\right) .
$$

For each $k$,

$$
\phi\left(\left\{\varepsilon_{1}\right\} \times \cdots \times\left\{\varepsilon_{k}\right\} \times\{0,1\} \times\{0,1\} \times \cdots\right)=C \cap N\left(\varepsilon_{1}, \ldots, \varepsilon_{k}\right),
$$

so that $\phi$ is indeed a homeomorphism. Let $E$ be the countable subset in $\{0,1\}^{\mathbb{N}}$ of sequences which eventually terminate in all zeros or all ones. Let $F=\{0,1\}^{\mathbb{N}} \backslash E$. Let $D=C \backslash \phi(E)=\phi(F)$. We now show that for any $(x, y) \in D, g_{i}(x, y) \in D$ for infinitely many $i$, positive and negative, and each such $i$ is even. Let $\left\{\varepsilon_{k}\right\}_{k=1}^{\infty}$ be a sequence of zeros and ones not in $E$ and let $(x, y)=\phi\left(\varepsilon_{1}, \varepsilon_{2}, \ldots\right)$, which is in $D$. Then $(x, y) \in N\left(\varepsilon_{1}, \ldots, \varepsilon_{k}\right)$ for 
every $k$. If $\varepsilon_{1}=0$, then $v(x, y) \notin C$, since it is not in $N_{0}$, but $h v(x, y) \in$ $N\left(1, \varepsilon_{2}, \ldots, \varepsilon_{k}\right)$ for all $k$ so that $h v(x, y)=\phi\left(1, \varepsilon_{2}, \varepsilon_{3}, \ldots\right) \in D$ and the index of $(x, y)$ with respect to $D$ is even. Assume now that $\varepsilon_{1}=1$ and let $p$ be the integer such that $\varepsilon_{1}=1, \ldots, \varepsilon_{p}=1, \varepsilon_{p+1}=0$, i.e., $p+1$ is the first integer with $\varepsilon_{p+1}=0$. Then $(x, y) \in N\left(\varepsilon_{1}, \ldots, \varepsilon_{p+1}, \varepsilon_{p+2}, \ldots, \varepsilon_{n}\right)$ for every $n \geq p+1$. Equivalently,

$$
(x, y) \in \tau^{n(1)+\cdots+n(p)} N\left(0, \ldots, 0_{p}, 0_{p+1}, \varepsilon_{p+2}, \ldots, \varepsilon_{n}\right) .
$$

Now for all $n \geq p+1$, the sets

$$
\begin{aligned}
& v \tau^{n(1)+\cdots+n(p)} N\left(0, \ldots, 0_{p}, 0_{p+1}, \varepsilon_{p+2}, \ldots, \varepsilon_{n}\right), \\
& h v \tau^{n(1)+\cdots+n(p)} N\left(0, \ldots, 0_{p}, 0_{p+1}, \varepsilon_{p+2}, \ldots, \varepsilon_{n}\right)
\end{aligned}
$$

and so on up to

$$
v \tau^{n(p+1)-1} N\left(0, \ldots, 0_{p}, 0_{p+1}, \varepsilon_{p+2}, \ldots, \varepsilon_{n}\right)
$$

are disjoint from $C$, hence also disjoint from $D$. On the other hand, for all $n \geq p+1$,

$$
\tau^{n(p+1)} N\left(0, \ldots, 0_{p+1}, \varepsilon_{p+2}, \ldots, \varepsilon_{n}\right)=N\left(0, \ldots, 0_{p}, 1_{p+1}, \varepsilon_{p+2}, \ldots, \varepsilon_{n}\right),
$$

i.e.,

$$
\begin{aligned}
\tau^{n(p+1)-(n(1)+\cdots+n(p))} \tau^{n(1)+\cdots+n(p)} N & \left(0, \ldots, 0_{p}, 0_{p+1}, \varepsilon_{p+2}, \ldots, \varepsilon_{n}\right) \\
& =N\left(0, \ldots, 0_{p}, 1_{p+1}, \varepsilon_{p+2}, \ldots, \varepsilon_{n}\right),
\end{aligned}
$$

i.e.,

$$
\begin{aligned}
& \tau^{n(p+1)-(n(1)+\cdots+n(p))} N\left(1, \ldots, 1_{p}, 0_{p+1}, \varepsilon_{p+2}, \ldots, \varepsilon_{n}\right) \\
&=N\left(0, \ldots, 0_{p}, 1_{p+1}, \varepsilon_{p+2}, \ldots, \varepsilon_{n}\right) .
\end{aligned}
$$

We thus see that if $i<2(n(p+1)-(n(1)+\cdots+n(p)))$ then $g_{i}(x, y) \notin D$, while if $i=2(n(p+1)-(n(1)+\cdots+n(p)))$ then

$$
\begin{aligned}
g_{i}(x, y) & =\bigcap_{n=p+1}^{\infty} N\left(0, \ldots, 0_{p}, 1_{p+1}, \varepsilon_{p+2}, \ldots, \varepsilon_{n}\right) \\
& =\phi\left(0, \ldots, 0_{p}, 1_{p+1}, \varepsilon_{p+2}, \ldots, \varepsilon_{n}, \ldots\right) \in D,
\end{aligned}
$$

which shows that the index of $(x, y)$ is even, being equal to $2(n(p+1)-$ $(n(1)+\cdots+n(p)))$.

Since a point $(x, y) \in D$ is $\phi\left(\varepsilon_{1}, \varepsilon_{2}, \ldots\right)$ for some $\left(\varepsilon_{1}, \varepsilon_{2}, \ldots\right) \in F$, and since zeros and ones occur infinitely often in this sequence, we see that there are infinitely many even positive integers $i$ with $g_{i}(x, y) \in D$. Further there are no odd $i$ with this property. Indeed, if $\varepsilon_{1}=0$ then the index of $(x, y)$ is $2 n(1)$, while if $\varepsilon_{1}=1$, then the index of $(x, y)$ is $2(n(p+1)-n(1)-n(2)-$ $\cdots-n(p))$, where $p$ is the first integer with $\varepsilon_{p+1}=0$. Since every point in $D$ is the image of a point in $D \cap N\left(0, \ldots, 0_{n}\right)$ under a suitable power of $\tau$ (which depends on $n$ ), we see that $g_{k}(x, y) \in D$ for infinitely many negative 
even integers $k$. It remains to show that $\Pi \cap D$ is not countably generated. Let $\sigma: F \rightarrow F$ be defined by

$$
\sigma\left(\varepsilon_{k}\right)_{k=1}^{\infty}=\left(0, \ldots, 0_{p}, 1, \varepsilon_{p+2}, \varepsilon_{p+3}, \ldots\right)
$$

where $p \geq 1$ is the first integer with $\varepsilon_{p+1}=0$. The invertible Borel map $\sigma$ is ergodic with respect to the Haar measure on $\{0,1\}^{\mathbb{Z}}$ restricted to $F$, so its orbits form a partition $\mathcal{Q}$ of $F$ which is not countably generated. Since $\Pi \cap D=\phi \mathcal{Q}, \Pi \cap D$ is not countably generated. This proves the theorem.

4.11. RemARK. Let $U$ be a good Borel set such that for each $(x, y) \in U$ there are points $\left(x, y^{\prime}\right),\left(x^{\prime}, y\right) \in U$ with $x \neq x^{\prime}, y \neq y^{\prime}$. By repeated use of the axiom of choice it is possible to construct a set $S \subset U$ (not necessarily Borel) with doubleton sections and intersecting each linked component of $U$. It is natural to ask if one can construct $S$ to be Borel measurable. In this connection we mention a result of L. Harrington, A. Kechris and A. Louveau [6] which says that if a Borel equivalence relation $\mathcal{L}$ on $[0,1]$ is not countably generated then there is a Borel subset $A$ of $[0,1]$ such that the partition $A \cap \mathcal{L}$ is isomorphic to the Vitali partition of $[0,1]$.

We close this section by giving a result on the existence of a Borel crosssection of the partition $\Pi$ of a good Borel set $S$ into its linked components. If the linked components are finite then, by 1.11, $\Pi$ admits a Borel crosssection; if the linked components are countable and $\Pi$ is countably generated then too $\Pi$ admits a Borel cross-section by 1.9.

4.12. TheOREM. Let $S$ be a good Borel set with sections $S_{x}, x \in X$, and $S^{y}, y \in Y$, countable. Suppose for every linked component $C$ there exists an $n$ such that every link contained in $C$ is of length at most $n$. Then $\Pi$ admits a Borel cross-section.

Proof. We shall define Borel sets $G_{n}, H_{n}, V_{n}, n=0,1, \ldots$, such that $\bigcup_{n=0}^{\infty}\left(G_{n} \cup H_{n} \cup V_{n}\right)$ will be a Borel cross-section of $\Pi$. Set $S_{0}=S$. Define

$$
G_{0}=\left\{(x, y):\left(S_{0}\right)_{x} \text { and }\left(S_{0}\right)^{y} \text { are both singletons }\right\} .
$$

By 1.12, $G_{0}$ is Borel. Note that $G_{0}$ is the union of those linked components of $S$ that are singletons. We define $H_{0}, V_{0}$ as follows: Set $T_{0}=S_{0} \backslash G_{0}$ and

$$
E=\left\{x \in X:\left(T_{0}\right)_{x} \text { is a singleton }\right\} .
$$

By $1.12, E$ is Borel. Let

$$
\begin{aligned}
T_{0}^{\prime} & =T_{0} \cap(E \times Y), \\
B & =\left\{\left((x, y),\left(x^{\prime}, y\right),\left(x^{\prime}, y^{\prime}\right)\right) \in T_{0}^{\prime} \times T_{0} \times T_{0}: x \neq x^{\prime}, y \neq y^{\prime}\right\}, \\
T_{0}^{\prime \prime} & =\left\{(x, y) \in T_{0}^{\prime}: \exists\left(x^{\prime}, y\right),\left(x^{\prime}, y^{\prime}\right) \in T_{0}\left(x \neq x^{\prime} \& y \neq y^{\prime}\right)\right\} .
\end{aligned}
$$

Since $S$ has countable sections, each $B_{(x, y)}$ is countable. Further, $T_{0}^{\prime \prime}$ is the projection of $B$. Therefore, by Lusin's theorem (1.8), $T_{0}^{\prime \prime}$ is Borel. Set $D=$ $T_{0}^{\prime} \backslash T_{0}^{\prime \prime}$. Note that $D$ is the union of all those linked components that are 
contained in a horizontal line. Since $D \subset S$, each $D_{y}$ is countable for all $y$. We take $H_{0}$ to be the graph of any Borel function from $\pi_{Y}(D)$ to $X$ which is contained in $D$. We define $V_{0}$ similarly except that we interchange the role of $X$ - and $Y$-coordinates.

Note that the union of $G_{0}, D$ and the union of all the linked components of $S$ that are contained in a vertical line is a Borel set, say $D_{0}$. Now set $R_{1}=S_{0} \backslash D_{0}$. Note that

$$
\pi_{X}\left(R_{1}\right) \cap \pi_{X}\left(D_{0}\right)=\emptyset=\pi_{Y}\left(R_{1}\right) \cap \pi_{Y}\left(D_{0}\right) .
$$

Also note that $R_{1}$ is Borel and it is the union of all those linked components $C$ of $S$ that contain at least one link of length 3 .

We next define $G_{1}, H_{1}, V_{1}$. Let

$$
\begin{aligned}
& A=\left\{x \in X:\left(R_{1}\right)_{x} \text { is a singleton }\right\}, \\
& B=\left\{y \in Y:\left(R_{1}\right)^{y} \text { is a singleton }\right\} .
\end{aligned}
$$

By 1.12, $A$ and $B$ are Borel sets. The set $R_{1} \cap((A \times Y) \cup(X \times B))$ is the set of all $(x, y) \in R_{1}$ that occur at the ends of a maximal link. Set $S_{1}=$ $R_{1} \backslash((A \times Y) \cup(X \times B))$. We define $G_{1}, H_{1}$ and $V_{1}$ from $S_{1}$ in the same way we defined $G_{0}, H_{0}$ and $V_{0}$ from $S_{0}$. Note that $\left(G_{0} \cup H_{0} \cup V_{0}\right) \cup\left(G_{1} \cup H_{1} \cup V_{1}\right)$ is a Borel set picking up exactly one point from all those linked components $C$ such that every link contained in $C$ is of length at most 4 .

Proceeding similarly we define $G_{n}, H_{n}, V_{n}$ for $n \geq 2$ by induction on $n$ such that for every $n, \bigcup_{i \leq n}\left(G_{i} \cup H_{i} \cup V_{i}\right)$ picks up exactly one point from all the linked components not containing any link of length greater than $2 n$. Since for every linked component $C$ of $S$ there exists an $n$ such that every link contained in $C$ is of length at most $n, \bigcup_{n=0}^{\infty}\left(G_{n} \cup H_{n} \cup V_{n}\right)$ will be a Borel cross-section of $\Pi$ and the proof is complete.

We do not know if the above result is true under the weaker assumption that $S$ has no infinite link.

\section{Sequentially good measures}

5.1. Definition. Let $m$ be a probability measure on Borel subsets of $X \times Y$, with $X, Y$ Polish, and call $m$ sequentially good if every bounded Borel function is a pointwise a.e. limit of a sequence $f_{n}, n=1,2, \ldots$, of functions of the form

$$
f_{n}(x, y)=u_{n}(x)+v_{n}(y)
$$

with $u_{n}, v_{n}$ Borel measurable but not necessarily bounded.

5.2. REMARK. This definition of sequentially good measure is weaker than the one given in [10] and seems more appropriate. The proof given in [10], that a sequentially good measure admits a good Borel set as support is valid with the new definition as well and we briefly recall this proof here. 
Let $S \subset X \times Y$ and let $\mathbb{A}$ be an algebra of complex-valued functions closed under conjugation, separating points and containing the constants. If every function $f$ in $\mathbb{A}$ is of the form

$$
f(x, y)=\lim _{n \rightarrow \infty}\left(u_{n}(x)+v_{n}(y)\right)
$$

then $S$ is good, for otherwise $S$ will admit a loop $L$ on which every function $\left.f \in \mathbb{A}\right|_{L}$ is of the form (2). Since $L$ is a finite set, by the Stone-Weierstrass theorem $\left.\mathbb{A}\right|_{L}$ coincides with all functions on $L$, and in the light of (2) above, $L$ is sequentially good, hence good. The contradiction shows that $S$ is a good set. This simple observation implies that a sequentially good measure admits a good Borel set $S$ as support. For if $m$ is sequentially good, we let $S$ be a Borel set of full measure on which

$$
I_{A}(x, y)=\lim _{n \rightarrow \infty}\left(u_{n}(x)+v_{n}(y)\right), \quad(x, y) \in S,
$$

holds for $A$ in a countable field $\mathcal{A}$ of Borel subsets of $X \times Y$ separating points. Then (3) will hold also for finite linear combinations of $I_{A}, A \in \mathcal{A}$, with complex coefficients. These functions form an algebra of functions closed under conjugation. Further they separate points and contain the constants. So $S$ is a good Borel set which supports $m$. We have proved:

5.3. THEOREM. If $m$ is sequentially good then $m$ admits a Borel support $S$ which is good.

5.4. EXAMPles. Any probability measure on the sets of Examples 1.4 and 1.5 is sequentially good (with $\theta, u$ irrational respectively). We prove this for the set $S$ of 1.5. A similar proof works for the other case.

Let $S_{n}=S \backslash C_{n}$, where $C_{n}$ is an open segment of $S$ of length $1 / n$ with $C_{n+1} \subset C_{n}$ for all $n$. Then $S_{n} \subset S_{n+1}$ with the union of $S_{n}$ 's equal to $S$ a.e. Let $g_{k}, k \in \mathbb{Z}$, be the enumeration of the group $\mathbb{G}$ as in Section 2.1. Since the $\mathbb{G}$-action is ergodic, for any $(x, y) \in S$ and any $n, g_{k}(x, y) \in C_{n}$ for infinitely many positive $k$ and for infinitely many negative $k$. This implies that linked components of $S_{n}$ have finite cardinality, so by 1.11 they admit a Borel cross-section. By 4.6 every Borel function on $S_{n}$ is of the form $u+v$ with $u, v$ Borel measurable. If $f$ is a bounded Borel function on $S$, then

$$
\left.f\right|_{S_{n}}(x, y)=u_{n}(x)+v_{n}(y), \quad(x, y) \in S_{n},
$$

with $u_{n}, v_{n}$ Borel measurable. Further

$$
\left.f\right|_{S_{n}}(x, y)=f(x, y) I_{S_{n}}(x, y), \quad(x, y) \in S_{n} .
$$

(There is a logical difference between the two sides since $\left.f\right|_{S_{n}}$ is defined on $S_{n}$ while $f I_{S_{n}}$ is defined on all of $S$.) So

$$
f I_{S_{n}}=u_{n}(x)+v_{n}(y), \quad(x, y) \in S_{n}
$$


and since $f I_{S_{n}}(x, y) \rightarrow f(x, y)$ for $(x, y) \in S$, we see that any probability measure on $S$ is sequentially good.

5.5. It is natural to ask whether any probability measure supported on a Borel set without loop is sequentially good and more strongly whether any bounded Borel function $f$ defined on such a set is of the form

$$
f(x, y)=\lim _{n \rightarrow \infty}\left(u_{n}(x)+v_{n}(y)\right), \quad(x, y) \in S,
$$

with $u_{n}, v_{n}$ Borel measurable.

\section{Connection with simplicial measures}

6.1. Let $X, Y$ be Polish spaces. A probability measure $\mu$ on $X \times Y$ (whose marginals are denoted by $\mu_{1}$ and $\mu_{2}$ ) is called simplicial if it is an extreme point of the convex set of all probability measures on $X \times Y$ whose marginals are the same as those of $\mu$. A basic theorem of Lindenstrauss states that a probability measure $\mu$ on $X \times Y$ is simplicial if and only if the collection of functions of the form

$$
f(x, y)=u(x)+v(y), \quad u \in L^{1}\left(X, \mu_{1}\right), v \in L^{1}\left(Y, \mu_{2}\right),
$$

is dense in $L^{1}(X \times Y, \mu)$.

It is clear from this that a simplicial measure is sequentially good, hence by 5.3 it admits a good Borel set as support.

6.2. It is easy to see that if $\lambda$ and $\nu$ are continuous probability measures on $[0,1]$ then any Borel set with positive $\lambda \times \nu$ measure admits a loop with four points. Indeed, if $A$ is such a set then there exist two distinct points $x$ and $x^{\prime}$ such that the sections $A_{x}=\{y:(x, y) \in A\}, A_{x^{\prime}}=\left\{y:\left(x^{\prime}, y\right)\right.$ $\in A\}$ intersect in a set of positive $\nu$-measure and since $\nu$ is continuous, we can find two distinct points $y, y^{\prime}$ in this intersection. Clearly the points $(x, y),\left(x, y^{\prime}\right),\left(x^{\prime}, y^{\prime}\right),\left(x,^{\prime} y\right)$ form a loop in $A$. Since a simplicial measure admits a good Borel support, hence a Borel set not admitting a loop, we see that a simplicial measure is singular to $\lambda \times \nu$, where $\lambda$ and $\nu$ are any continuous probability measures on $X$ and $Y$ respectively.

This result, due to Štěpán [20], is a strengthening of a similar result due to Lindenstrauss [13], but their proofs are more involved.

6.3. A Borel set $E \subset X \times Y$ is called a set of marginal uniqueness (briefly an MU-set) if every probability measure $\mu$ supported on $E$ is an extreme point of the convex set of all probability measures on $X \times Y$ with marginals same as those of $\mu$. Clearly any Borel subset of an MU-set is an MU-set, and since a loop is not an MU-set, we see that an MU-set cannot contain a loop. Therefore an $M U$-set is a good set. Further, if $S$ is a good Borel set whose linked components admit a Borel cross-section then $S$ is an MU-set. 
To see the latter, let $C$ be a Borel cross-section of the linked components of $S$ and let $S_{n}$ be the points $(x, y) \in S$ linked to a point in $C$ by a path of length $\leq n$. Then $S_{n} \subset S_{n+1}$ and $\bigcup_{n=1}^{\infty} S_{n}=S$. For any bounded Borel measurable function $f$ on $S, f I_{S_{n}} \rightarrow f$ in the $L^{p}$ norm of any probability measure on $S, 1 \leq p<\infty$. Moreover if $v_{n}$ is chosen to vanish on the $y$ coordinates of points in $C$, then $u_{n}, v_{n}$ in the solution of $f I_{S_{n}}=u_{n}+v_{n}$ are bounded (by the Remark in 3.2), hence in $L^{p}, 1 \leq p<\infty$, of any probability measure on $S$. Clearly, then, the functions of the form $u(x)+v(y)$ with $u, v$ bounded and measurable are dense in $L^{p}, 1 \leq p<\infty$, of any probability measure on $S$. In particular this holds for $p=1$. the By Lindenstrauss' theorem we see that $S$ is an MU-set (see also [7, Theorem 20]).

6.4. For a probability measure $\mu$ on $X \times Y$ with marginals $\mu_{1}$ and $\mu_{2}$,

$$
\begin{aligned}
\left(L^{1}\left(X, \mu_{1}\right)+L^{1}\left(Y, \mu_{2}\right)\right)^{\perp} & =\left\{\phi \in L^{\infty}(X \times Y, \mu):\right. \\
\int_{X \times Y} f \phi d \mu & \left.=\int_{X \times Y} g \phi d \mu=0, f \in L^{1}\left(X, \mu_{1}\right), g \in L^{1}\left(Y, \mu_{2}\right)\right\} .
\end{aligned}
$$

This shows that

$$
\begin{aligned}
\left(L^{1}\left(X, \mu_{1}\right)+L^{1}\left(Y, \mu_{2}\right)\right)^{\perp}=\left\{\phi \in L^{\infty}(X \times Y, \mu):\right. & \\
& \left.\phi_{+} d \mu, \phi_{-} d \mu \text { have the same marginals }\right\} .
\end{aligned}
$$

(In case $\mu$ is simplicial, the left hand side, hence the right hand side, vanishes.)

Now assume that $\mu$ is supported on a good Borel set $S$ with doubleton sections. If $f \in L^{1}(X \times Y, \mu)$, then $f+f \circ v \in L^{1}\left(X, \mu_{1}\right), f+f \circ h \in L^{1}\left(Y, \mu_{2}\right)$. If $\phi$ is in $\left(L^{1}\left(X, \mu_{1}\right)+L^{1}\left(Y, \mu_{2}\right)\right)^{\perp}$, then

$$
\int_{X \times Y} f \phi d \mu=-\int_{X \times Y} f(\phi d \mu) \circ v, \quad \int_{X \times Y} f \phi d \mu=-\int_{X \times Y} f(\phi d \mu) \circ h,
$$

whence $\phi_{+} d \mu, \phi_{-} d \mu$ are $\mathbb{G}_{0}$-invariant and they can be distinct only if the $\mathbb{G}_{0}$-action admits two distinct invariant probability measures. These observations and those of 2.6 allow us to conclude: MU-set;

(i) if there is no $\mathbb{G}$-invariant probability measure on $S$ then $S$ is an

(ii) if the $\mathbb{G}$-action on $S$ is uniquely ergodic then $S$ is an MU-set if and only if $\mathbb{G}_{0}$ is also uniquely ergodic;

(iii) finally, decomposing $S$ into $\mathbb{G}$-invariant Borel sets on each of which the $\mathbb{G}$-action is uniquely ergodic, we see that $S$ is an MU-set if and only if each $\mathbb{G}$-invariant uniquely ergodic component of $S$ is also $\mathbb{G}_{0}$-ergodic.

This completely describes the Borel sets with doubleton sections which are also MU-sets. 
6.5. Now let $S$ be a compact subset of the unit square, let $C(S)$ denote the space of continuous complex-valued functions on $S$, and let $C_{1}$ and $C_{2}$ denote the subspaces of continuous functions which depend on $x$ alone and $y$ alone respectively. The question as to when $C_{1}+C_{2}$ is dense in $C$ in the uniform norm is discussed in $[15,16]$. It is clear that this holds if and only if $\left(C_{1}+C_{2}\right)^{\perp}$ is empty. This in turn holds if and only if $S$ is an MU-set. So, by 6.3 , if $S$ is good and the linked components of $S$ admit a Borel cross-section then $C_{1}+C_{2}$ is dense in $C(S)$ in the uniform norm. In [15] this is proved under the assumption that $S$ has no loops and the linked components are compact, a condition which implies that the linked components admit a Borel cross-section (see 1.11).

For compact MU-sets it is clear that $L^{p}\left(X, \mu_{1}\right)+L^{p}\left(Y, \mu_{2}\right)$ is dense in $L^{p}(S, \mu), 1 \leq p<\infty$, where $\mu$ is any probability measure on $S$. Finally, if $S$ is a compact set with doubleton sections then $C_{1}+C_{2}$ is dense in $C(S)$ under uniform norm if and only if every $\mathbb{G}$-ergodic probability measure on $S$ is also $\mathbb{G}_{0}$-ergodic.

\section{References}

[1] V. Beneš and J. Štěpán, The support of extremal probability measures with given marginals, in: Mathematical Statistics and Probability Theory, Vol. A, M. L. Puri et al. (eds.), Reidel, Dordrecht, 1987, 33-41.

[2] - - - Extremal solutions in the marginal problem, in: Advances in Probability Distributions with Given Marginals, Math. Appl. 67, Dordrecht, 1991, 189-207.

[3] R. C. Cowsik, A. Kłopotowski and M. G. Nadkarni, When is $f(x, y)=u(x)+v(y)$ ?, Proc. Indian Acad. Sci. Math. Sci. 109 (1999), 57-64.

[4] A. Diego and A. Germani, Extremal measures with prescribed marginals (finite case), J. Combin. Theory Ser. A 13 (1972), 353-366.

[5] E. Effros, Transformation groups and $C^{*}$-algebras, Ann. of Math. 81 (1965), 38-55.

[6] L. A. Harrington, A. S. Kechris and A. Louveau, A Glimm-Effros dichotomy for Borel equivalence relations, J. Amer. Math. Soc. 3 (1990), 202-228.

[7] K. Hestir and S. Williams, Supports of doubly stochastic measures, Bernoulli 1 (1995), 217-243.

[8] J. Kaniewski and C. A. Rogers, Double uniformization, J. London Math. Soc. (2) 22 (1980), 521-533.

[9] A. S. Kechris, Classical Descriptive Set Theory, Grad. Texts in Math. 156, Springer, New York, 1995.

[10] A. Kłopotowski and M. G. Nadkarni, Shift invariant measures and simple spectrum, Colloq. Math. 84/85 (2000), 385-394.

[11] D. König, Sur les correspondances multivoques des ensembles, Fund. Math. 8 (1926), 114-134.

[12] M. Laczkovich, Closed sets without measurable matchings, Proc. Amer. Math. Soc. 103 (1988), 894-896.

[13] J. Lindenstrauss, A remark on doubly stochastic measures, Amer. Math. Monthly 72 (1965), 379-382. 
[14] V. Losert, Counter-examples of some conjecture about doubly stochastic measures, Pacific J. Math. 99 (1982), 387-397.

[15] D. Marshall and A. O'Farrell, Uniform approximation by real functions, Fund. Math. 104 (1979), 203-211.

[16] - - - Approximation by a sum of two algebras. The lightning bolt principle, J. Funct. Anal. 52 (1983), 353-368.

[17] M. G. Nadkarni, Basic Ergodic Theory, 2nd ed., Hindustan Book Agency, New Delhi, and Birkhäuser, Basel, 1998.

[18] G.-C. Rota and L. H. Harper, Matching theory. An Introduction, in: Adv. Probab. Related Topics 1, P. Ney (ed.), Dekker, New York, 1971, 169-215.

[19] S. M. Srivastava, A Course on Borel Sets, Grad. Texts in Math. 180, Springer, New York, 1998.

[20] J. Štěpán, Simplicial measures and sets of uniqueness in the marginal problem, Statist. Decisions 11 (1993), 289-299.

[21] B. Weiss, Measurable dynamics, in: Contemp. Math. 26, Amer. Math. Soc., 1984, 395-432.

Institut Galilée

Université Paris XIII

93430 Villetaneuse Cedex, France

E-mail: klopot@math.univ-paris13.fr

Stat-Math Unit

Indian Statistical Institute

203 B. T. Road

Calcutta, India 700035

E-mail: haimanti@isical.ac.in

smohan@isical.ac.in
Department of Mathematics University of Mumbai Kalina, Mumbai, India 400098 E-mail: nadkarni@math.mu.ac.in

Received 1 August 2000;

in revised form 27 July 2001 and 20 December 2001 